\title{
Decoherence in two-dimensional quantum random walks with traps
}

\author{
Meltem Gönülol, ${ }^{1}$ Ekrem Aydiner, ${ }^{2, *}$ and Özgür E. Müstecaplıŏlu ${ }^{3}$ \\ ${ }^{1}$ Department of Physics, Dokuz Eylül University, 35160 Izmir, Turkey \\ ${ }^{2}$ Department of Physics, Istanbul University, 34134 Istanbul, Turkey \\ ${ }^{3}$ Department of Physics, Koç University, Sariyer, 34450 Istanbul, Turkey
}

(Received 24 June 2009; published 27 August 2009)

\begin{abstract}
Quantum random walk in a two-dimensional lattice with randomly distributed traps is investigated. Distributions of quantum walkers are evaluated dynamically for the cases of Hadamard, Fourier, and Grover coins, and quantum to classical transition is examined as a function of the density of the traps. It is shown that traps act as a serious and additional source of quantum decoherence. Furthermore, nontrivial temporal dependence of the standard deviation of the probability distribution of the walker is found when the trapping imperfections are introduced.
\end{abstract}

DOI: $10.1103 /$ PhysRevA.80.022336

PACS number(s): 03.67.Lx, 05.40.Fb, 03.65.Yz

\section{INTRODUCTION}

A simple stochastic process can be introduced by the motion of a particle that can move in certain directions with some probabilities such that progress of the particle is independent of its preceding movements. Finding the spatial probability distribution of the particle, starting such a randomized motion from a given location is the statement of the so-called random walk problem. Random walk and its more generalized extensions have been used as simple models for many physical systems, in particular in solid-state physics and in astronomy [1], and in polymer models [2]. Classical random walks have found variety of applications in other fields, such as in economics and in computational sciences, as well. In particular, powerful randomized algorithms, especially for graph connectivity, satisfiability, probability amplification, [3] and Markovian chain simulations [4], have been developed based on classical random walks.

Quantum computation and quantum information are one of the most active research fields nowadays [5]. A quantum computer would be capable to run quantum algorithms, such as Deutsch-Jozsa [6], Shor's [7], or Grover's algorithm [8], much faster than any classical algorithms running on classical computers. One direction of research aiming to develop more quantum algorithms is to implement some classical algorithms directly by considering their quantum analogs. In this respect, quantum analogs of random walks, the so-called quantum random walks (QRWs) have received much attention recently (see, e.g., Refs. [9-11]). Some new quantum algorithms based on QRWs have already been proposed [12-15]. It is proven that a discrete time quantum walk can be used for searching unsorted database with a quadratic speedup [13], while the continuous time quantum walks can be exploited for traversing certain graphs exponentially faster than any classical algorithm [12].

There are various schemes and systems including ion traps [16], optical lattices [17,18], cavity QED [19], optical cavity [20], and linear optics $[21,22]$ that have been considered for practical realization of QRWs. Using nuclear-

\footnotetext{
*ekrem.aydiner@istanbul.edu.tr
}

magnetic resonance two- and three-qubit quantum information processors, QRW has been demonstrated on a square for its continuous time [23] and discrete time [24] versions. Such direct implementations of QRW are not essential for quantum algorithm developments. As long as there exists a quantum computer, QRW and associated quantum algorithm can always be realized on it. On the other hand, QRWs provide deep insight into transition to classical behavior out of a quantum behavior as well as into decoherence and coherent control of quantum systems. Rich quantum dynamics available within QRWs makes them appealing systems per se. A well-known property of a quantum walker is that it is more delocalized than a classical walker such that a quantum walker spreads out quadratically faster in time than a classical walker.

Quantum walkers are highly delocalized particles, and their QRW is very sensitive to decoherence. The rapid transition of QRW to a classical walk may limit their implementations and applications, yet at the same time, small amount of decoherence can be beneficial to speed up quantum algorithms based on QRWs [25]. In order to develop optimized quantum walks on noisy quantum channels, it is necessary to investigate decoherence mechanisms and characterize their effects on the propagation of a quantum walker. Decoherence in QRW can be discussed in general terms (for a review, see Ref. [26]) as well as in more specialized contexts, depending on a particular implementation. For that aim, QRWs on a line under various decoherence mechanisms, such as random measurements or broken links [27], and under various environmental noises, such as phase flip, bit flip, and generalized amplitude damping channels [28], have been examined. The general purpose of these works is to understand and resolve the different decoherence problems to be encountered in various one-dimensional implementations of QRWs. In this work we contribute the same purpose by considering an additional decoherence mechanism and by focusing on different QRWs in two dimensions. Keeping the walkers on the lines or planes of interest can be nontrivial and practical realizations of QRWs may face a serious problem of loss of walkers during the evolution. In this work, we consider loss of walkers as a decoherence mechanism.

A natural imperfection that can cause nonunitary evolution of a quantum walker would be loss of the walker at 
certain places along the propagation directions. This may correspond to absorption of photon in optical implementations, loss of atom due to thermal fluctuations or collisions in atom traps or in optical lattices, or immobilization of quasiparticles in solids and so on. Indeed, in the case of classical RW problem, the possibility of the trapping of classical random walks by randomly distributed traps on a lattice has been extensively studied using both theoretical and Monte Carlo methods [29-35]. The exact source of the loss of the quantum walker would depend on a particular implementation. In certain systems, such as optical lattices, imperfections can be introduced on purpose and controllably such as by adjusting the depths of the atom traps or by optical addressing of a set of lattice sites. The results to be reported in this paper are a systematic characterization of effects of immobilizing centers on the QRW and are independent of a specific implementation.

QRW in higher dimensions, especially in two dimensions, have been studied extensively [36,37]. In higher dimensions, decoherence effect can be of different significance than in one dimension $[38,39]$. In this paper, we numerically investigate the effect of decoherence that causes quantum to classical transition in a two-dimensional (2D) random walk with traps. Typical quantum walks, namely, Hadamard $(H)$, Fourier $(F)$, and Grover $(G)$ walks are considered. Their relative endurances against decoherence are characterized. Traps along the paths of the walkers are recognized as a serious source of decoherence that should be reckoned with for a potential implementation of the quantum walk, in addition to broken links or random measurements.

The paper is organized as follows. We first present a short review of the theory of the two-dimensional QRW together with our model of walk on a lattice with traps in Sec. II. Results of the numerical simulations and their discussions are given in Sec. III. Finally, in Sec. IV, we summarize the conclusions of this work.

\section{QUANTUM RANDOM WALKS IN A 2D LATTICE WITH TRAPS}

We consider a QRW in a two-dimensional lattice with traps. The traps at a given density are randomly distributed before the walk is started then the trap locations remain frozen. For a QRW on an infinite two-dimensional lattice, the coin space is $\mathcal{H}_{4}$, spanned by the basis states $\{|j, k\rangle, j, k$ $\in\{0,1\}\}$, and the position space is $\mathcal{H}_{\infty}$, spanned by the basis states $\{|m, n\rangle, m, n$ integers $\}$. The state of a quantum walker at time $t$ in $\mathcal{H}_{4} \otimes \mathcal{H}_{\infty}$ space is given by

$$
|\psi(t)\rangle=\sum_{j, k=0}^{1} \sum_{n, m=-\infty}^{\infty} A_{j k m n}(t)|j, k\rangle|m, n\rangle .
$$

One step of the quantum walker is defined by the unitary operator $U$ such that

$$
U=S\left(I_{4} \otimes C\right),
$$

where $I_{4}$ is the $4 \times 4$ identity matrix, $C$ is the coin operator which written as

$$
C=\sum_{j, k=0}^{1} \sum_{j^{\prime}, k^{\prime}=0}^{1} C_{j k j^{\prime} k^{\prime}}|j, k\rangle\left|j^{\prime}, k^{\prime}\right\rangle,
$$

and $S$ is the shift operator described by

$$
S|j, k\rangle|m, n\rangle=|j, k\rangle\left|m+(-1)^{j}, n+(-1)^{k}\right\rangle .
$$

We analyze the results for three different coins which are Hadamard, Fourier, and Grover. $H, F$, and $G$ coins in two dimensions are, respectively, given by

$$
\begin{aligned}
C_{H} & =\frac{1}{2}\left(\begin{array}{cccc}
1 & 1 & 1 & 1 \\
1 & -1 & 1 & -1 \\
1 & 1 & -1 & -1 \\
1 & -1 & -1 & 1
\end{array}\right), \\
C_{F} & =\frac{1}{2}\left(\begin{array}{cccc}
1 & 1 & 1 & 1 \\
1 & i & -1 & -i \\
1 & -1 & 1 & -1 \\
1 & -i & -1 & i
\end{array}\right), \\
C_{G} & =\frac{1}{2}\left(\begin{array}{cccc}
-1 & 1 & 1 & 1 \\
1 & -1 & 1 & 1 \\
1 & 1 & -1 & 1 \\
1 & 1 & 1 & -1
\end{array}\right) .
\end{aligned}
$$

The quantum walker begin to walk at $(0,0)$ point in the two-dimensional lattice. The initial states for Hadamard, Fourier, and Grover walks were chosen to guarantee a maximum spreading when the walk starts at the origin [40], and as such for the $H$, the $F$, and the $G$ coins, they are, respectively, taken to be as

$$
\begin{gathered}
|\psi(0)\rangle_{H}=\frac{1}{2}(|00\rangle+i|01\rangle-i|10\rangle+|11\rangle)|0,0\rangle \\
|\psi(0)\rangle_{F}=\frac{1}{2}\left[\left(|00\rangle+\frac{1-i}{\sqrt{2}}|01\rangle+|10\rangle-\frac{1-i}{\sqrt{2}}|11\rangle\right)|0,0\rangle\right] \\
|\psi(0)\rangle_{G}=\frac{1}{2}[(|00\rangle-|01\rangle-|10\rangle+|11\rangle)|0,0\rangle]
\end{gathered}
$$

Applying $U$ on the state of the walker (1), the relation that determines the spatiotemporal evolution of the quantum walker is obtained to be as

$$
A_{j k m n}(t+1)=\sum_{j^{\prime}, k^{\prime}=0}^{1} C_{j k j^{\prime} k^{\prime}} A_{j^{\prime} k^{\prime} m_{j} n_{j}}(t),
$$

with $m_{j}=m-(-1)^{j}$ and $n_{j}=n-(-1)^{k}$. With the amplitude of new positions of the quantum walker given by Eq. (11), the probability distribution of the walker at position $|m, n\rangle$ at time $t$ is determined by

$$
P_{m, n}(t)=\sum_{j, k=0}^{1}\left|A_{j, k ; m, n}(t)\right|^{2} .
$$



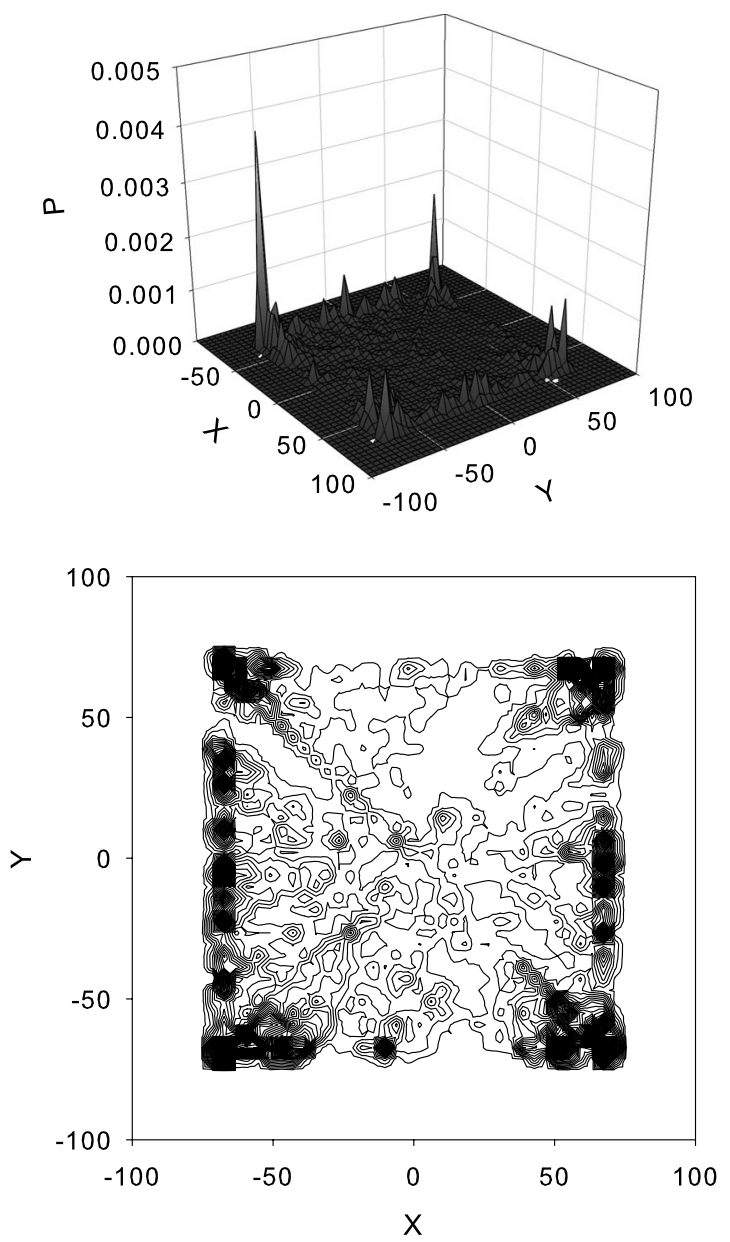

FIG. 1. The probability distribution of the Hadamard walk for $p=0.01$ after 100 iterations.

In the calculations, we assume that the traps are completely absorbing, so that if the quantum walker falls into a trap, it is annihilated with vanishing probability amplitudes at any later time. In the next section, the results of probability distributions obtained by numerical simulations for the cases of Hadamard, Fourier, and Grover walks with different trap densities will be presented. In particular, the examination of the standard deviation $\sigma$ will be used to characterize quantum to classical transition of the walks. In the case of a classical walk, $\sigma \propto \sqrt{t}$, while a quadratic gain in the spread is achieved in a quantum walk for which $\sigma \propto t$ due to quantum coherence. It is known that unitary noises such as broken links or nonunitary disturbances such as random measurements destroy this gain and make the quantum walk a classical one. Here, we explore how the traps, causing nonunitary evolution of the quantum walker in the two-dimensional lattice, might suppress the benefit of the quantum walk. As the traps are randomly distributed, it is necessary to consider many different initial configurations of the traps, so that we calculate

$$
\langle\sigma\rangle=\frac{1}{M} \sum_{r=1}^{M} \sigma_{r},
$$

where $M$ denotes the number of different configurations of random traps.
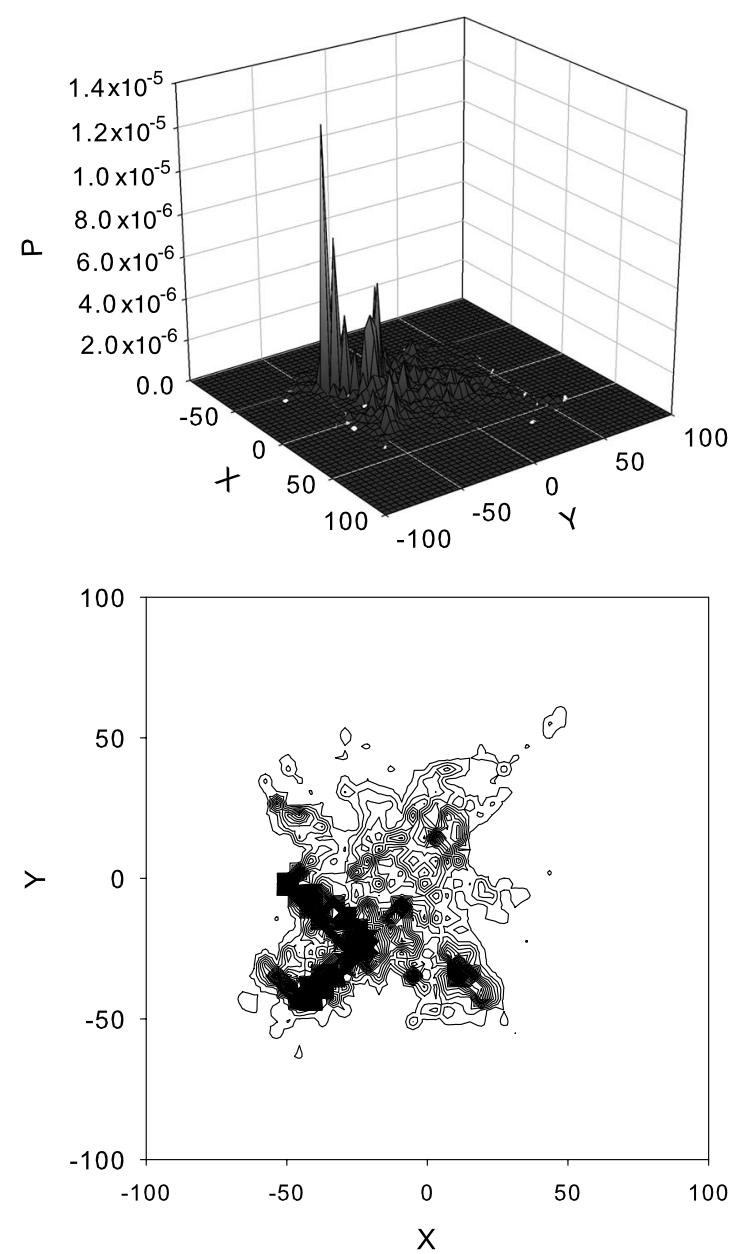

FIG. 2. The probability distribution of the Hadamard walk for $p=0.1$ after 100 iterations.

\section{RESULTS AND DISCUSSIONS}

In our simulations we have used an ensemble of 250 different configurations of randomly placed traps and the ensemble averaged results are presented in the figures for the calculated variance. We first investigate the case of a Hadamard walk. Quantum walker starts at the origin and its walk is numerically simulated for various trap densities. Figure 1 shows the probability distribution of the walker at time $t$ $=100$ for a trap density of $p=0.01$. The walk is seen to be still retaining its quantum character. One would expect a symmetric centrally peaked Gaussian-like distribution about the origin in the classical walk. We have found that such a behavior emerges and the walk becomes classical for about $p=0.1$ at $t=100$, which is shown in Fig. 2 .

The time dependence of the standard deviation for the Hadamard walk for different trap densities is plotted in Fig. 3 with a log-log scale. When there are no traps, $p=0$, the highest spatial spread of the walkers or the most quantum behavior is available. When trap densities increase, the standard deviation is dramatically altered. For small amount of traps, the spatial spread is gradually decreased but still $\sigma$ remains larger than the classical one, $\sigma_{c l}$, and grows faster. However, even when a modest amount of traps are introduced, the temporal behavior is no longer linear and $\sigma$ can grow as slow 


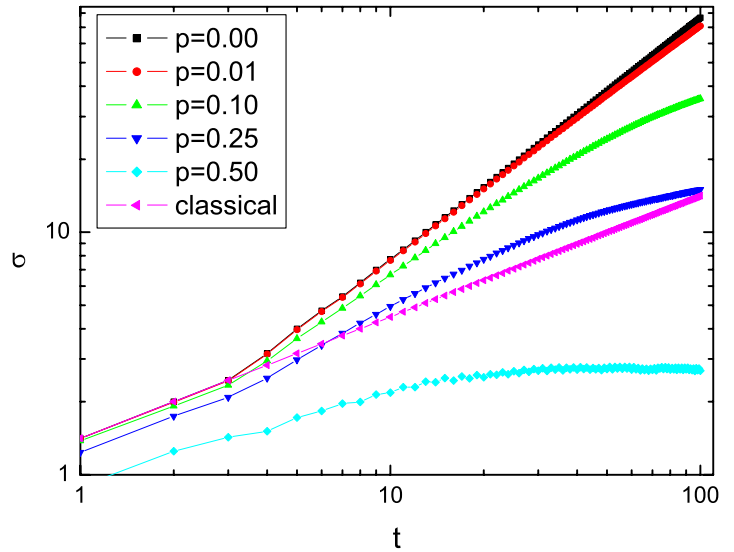

FIG. 3. (Color online) Time dependence of the standard deviation of the Hadamard walk in 2D trapping lattice with trap densities $p=0, p=0.01, p=0.1, p=0.25, p=0.5$, and classical random walk in 2D lattice.

as-or even slower-than $\sigma_{c l}$. This is in stark contrast with the other types of decoherence sources, such as broken links [39], for which simple power laws describe the time dependence of $\sigma$. The emergence of the nonlinear behavior of $\sigma$ in a trapped lattice can be attributed to the fact that the walkers
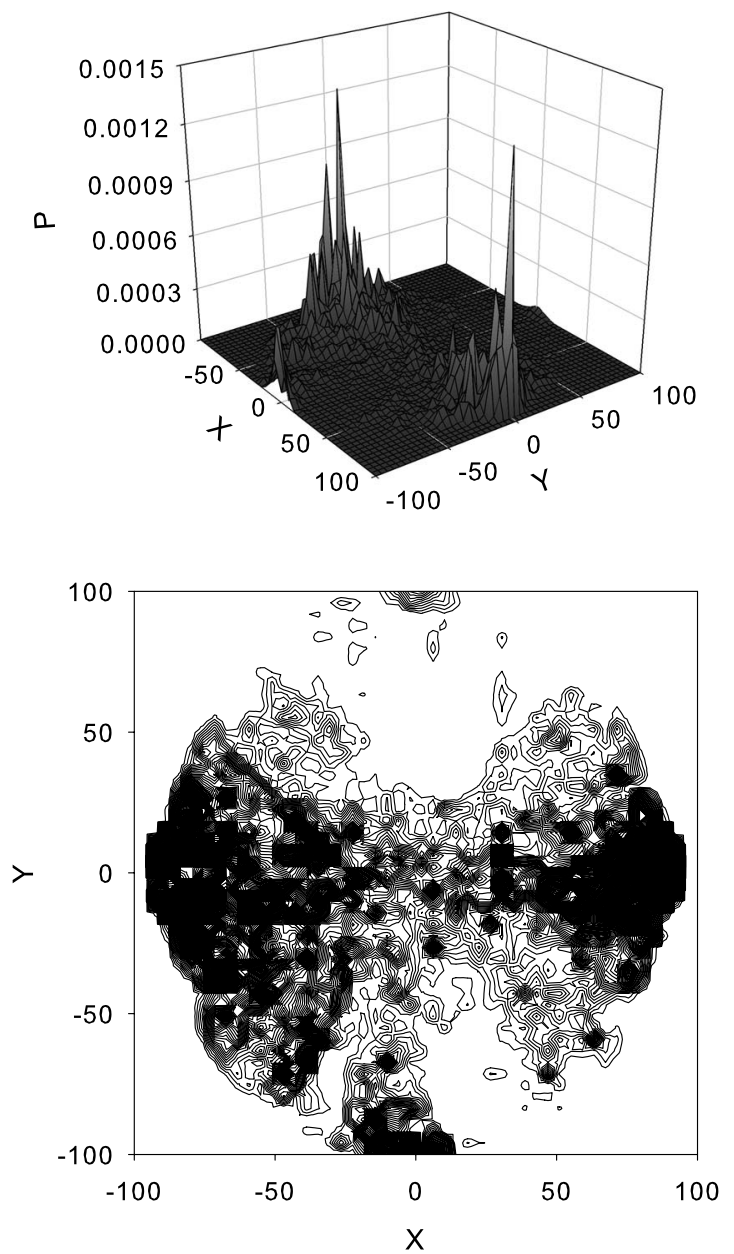

FIG. 4. The probability distribution of the Fourier walk for $p$ $=0.01$ after 100 iterations.
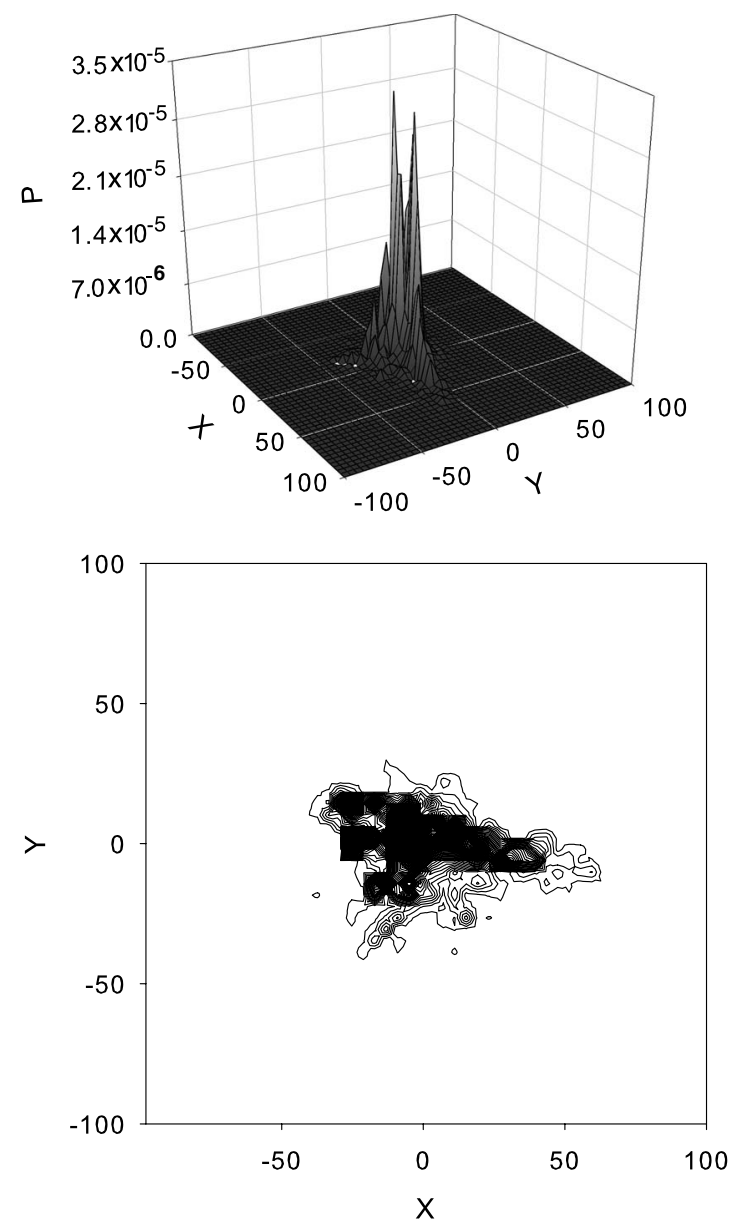

FIG. 5. The probability distribution of the Fourier walk for $p$ $=0.1$ after 100 iterations.

can survive in a trapped lattice only for a time and their probability of survival is related to the Kohlrausch-WilliamsWatts functions (stretched exponentials) of the form $\exp (-t / \tau)^{\beta}$ with $0<\beta<1$ as the stretching exponent and $\tau$ as a characteristic relaxation time [41-43].

When most of the lattice is trapped randomly, so that $p$ $>0.5$, the walkers become highly localized with $\sigma$ being significantly smaller than $\sigma_{c l}$. In other sources of decoherence, when random events are as frequent, $\sigma$ can become less than $\sigma_{c l}$, too [39]. However, traps force a more severe reduction which increases in time. Although $\sigma$ can be smaller in value than the classical one, it cannot grow slower than the classical walk in broken link disturbances. With the traps, however, both the magnitude of $\sigma$ and its growth rate can be made smaller than those of the classical walk. By examining the slopes of the curves in Fig. 3, it can be deduced that the transition from the quantum to the classical behavior happens at a time $\tau_{\text {decoh }} \approx 5 / p$, which is the decoherence time. After that, the growth rate of $\sigma$ gradually becomes smaller than the classical one, which is a reflection of the relatively short survival time and a stretched exponential behavior of the survival probability. In the broken links caused decoherence $\tau_{\text {decoh }} \approx 3 / p$ is found [39]. This suggests that twodimensional lattices can endure only slightly longer to decoherence due to traps. 


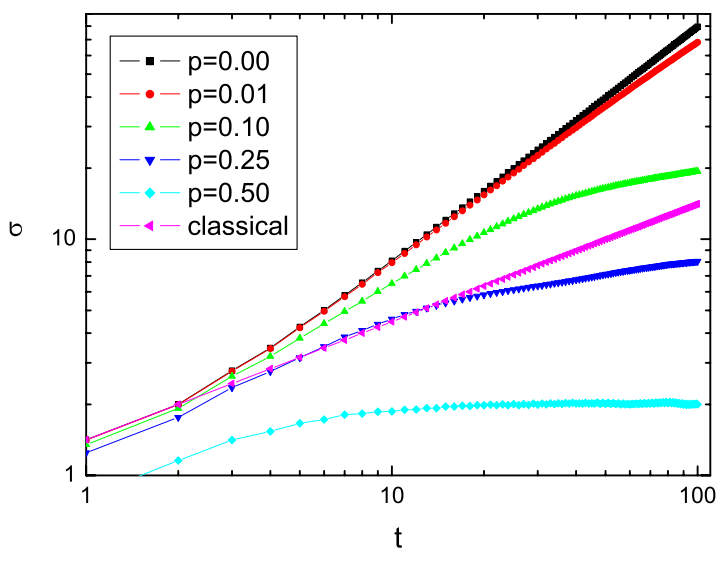

FIG. 6. (Color online) Time dependence of the standard deviation of the Fourier walk in $2 \mathrm{D}$ trapping lattice with trap densities $p=0, p=0.01, p=0.1, p=0.25, p=0.5$, and classical random walk in 2D lattice.

Let us now look at the other typical quantum walks. The probability distribution of the walker in the Fourier walk when the trap density is $p=0.01$ is given in Fig. 4 at iteration $t=100$. Analogous to the Hadamard walk, a significant quantum behavior is maintained at such low trap densities, while
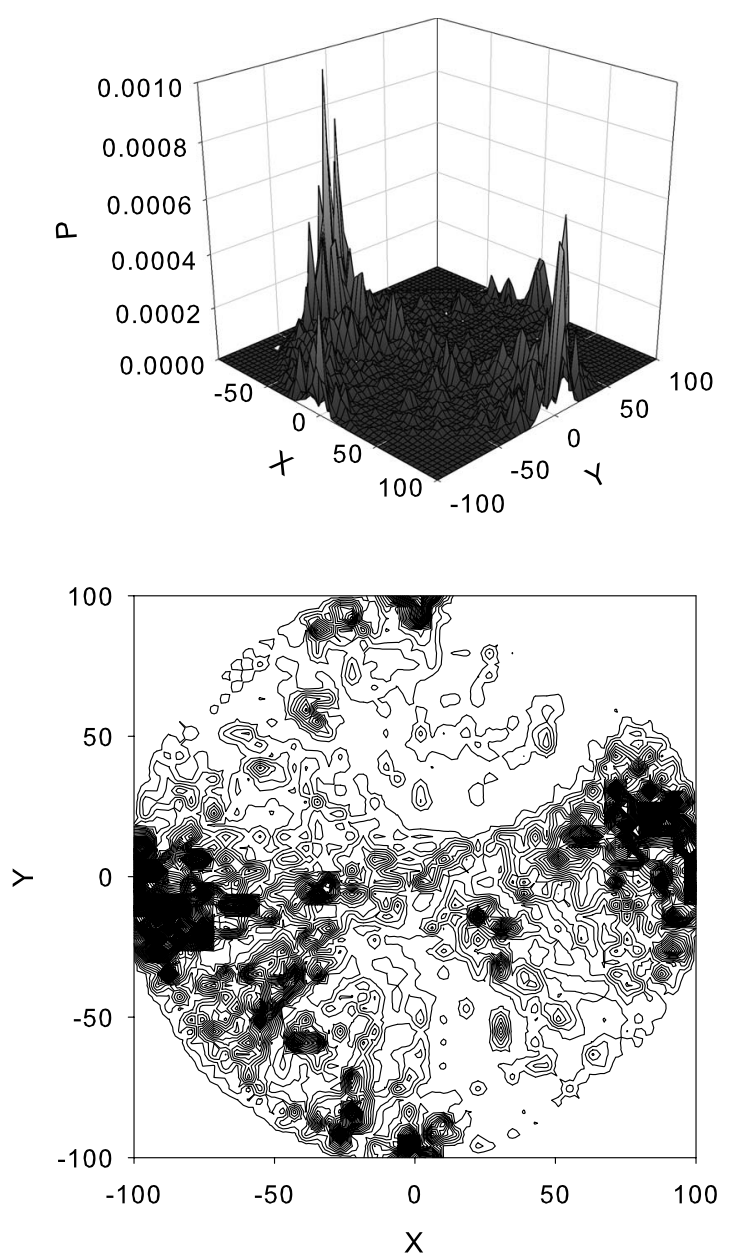

FIG. 7. The probability distribution of the Grover walk for $p$ $=0.01$ after 100 iterations.
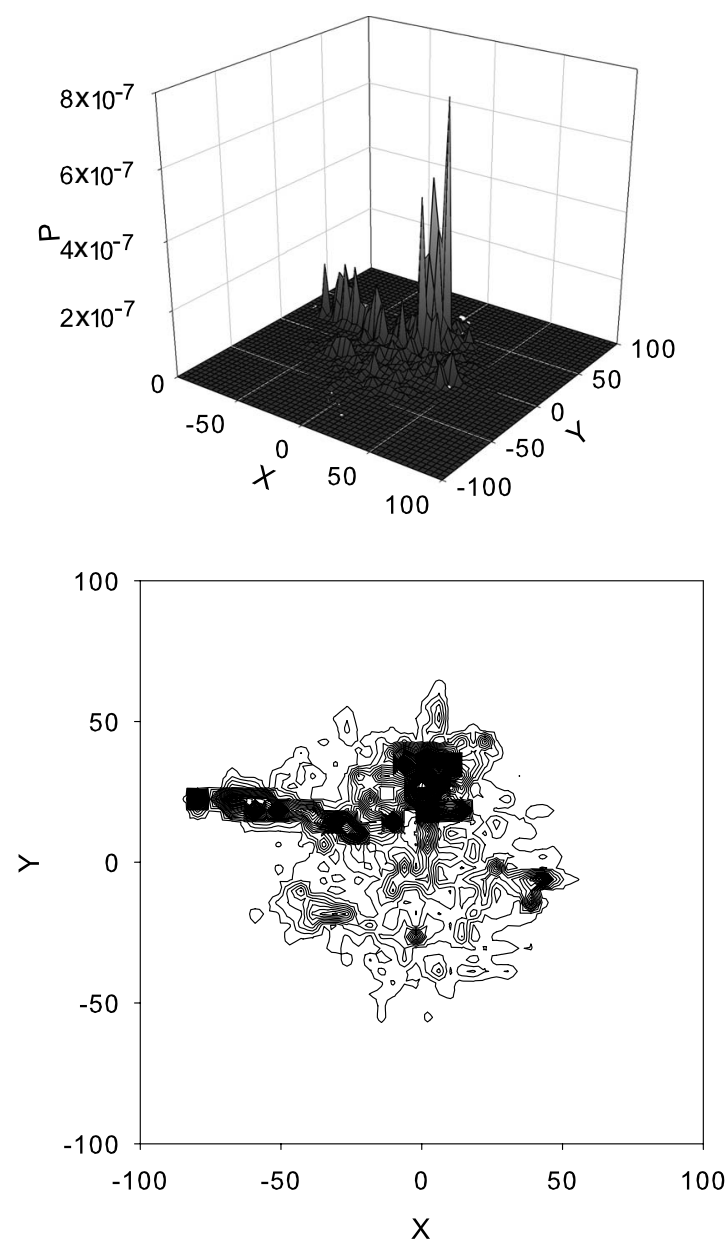

FIG. 8. The probability distribution of the Grover walk for $p$ $=0.1$ after 100 iterations.

as shown in Fig. 5 for $p=0.1$ and $t=100$ the classical behavior is emerged.

The time evolution of the standard deviation for the Fourier walk for different trap densities is depicted in Fig. 6 with a log-log scale. A strong nonlinear behavior is found in this case as in the Hadamard walk. When Fig. 6 is compared to Fig. 3, it is seen that the Fourier walk becomes classical more quickly than the Hadamard walk. This observation is in agreement with the broken links case [39].

Finally, we look at the case of the Grover walk for which Fig. 7 describes the probability distribution at time $t=100$ for the case of a trap density $p=0.01$, and Fig. 8 for $p=0.1$. The low and the high trap density behaviors lead to the similar conclusions as in the Grover and the Fourier walks, while the time dependence of the standard deviation in Fig. 9 indicates that the Hadamard walk comes to the classical behavior more slowly than the Fourier and the Grover walks. The Hadamard walk in two-dimensional lattice can be said to be more durable in decoherence due to traps while the Fourier is the least durable. This observation is also valid for the case of decoherence due to broken links [39], which is in fact a fundamentally based on symmetry properties of the probability distributions [39] when there is no decoherence and thus should be valid for any type of imperfections introduced. 


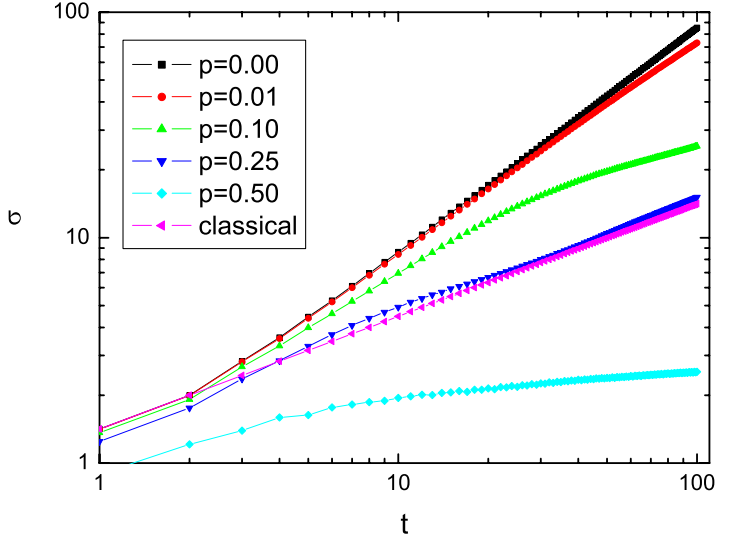

FIG. 9. (Color online) Time dependence of the standard deviation of the Grover walk in 2D trapping lattice with trap densities $p=0, p=0.01, p=0.1, p=0.25, p=0.5$, and classical random walk in 2D lattice.

\section{CONCLUSIONS}

Introducing traps along the path of a random walker has been investigated in detail classically in the context of the survival probability of particles in solid-state systems with defects and impurities. We examine this problem for the case of quantum random walk and show that traps act as a significant source of quantum decoherence and make the walk a classical one even when a modest number of traps are present. We consider the cases of three different quantum coins, which are Hadamard, Fourier, and Grover. The corresponding initial states for each coin are chosen to guarantee a maximum spreading when the walk starts at the origin. Characterization of the traps as a source of decoherence reveals that trapping forces transition to a classical walk from a quantum walk in a relatively longer time in comparison to the broken links. While all three types of the quantum walks become classical after a time decreasing with the trap density, it is found that quantum walk with a Hadamard coin is found to be the most tolerant to the density of the traps. A quantum walker with a Fourier coin would exhibit the lowest endurance against traps. Major difference in a trapped lattice is the survival time of the walkers that causes slower growth rates of the standard deviations than classical walks and lack of simple power-law temporal growth of the standard deviations due to the stretched exponential behavior of the survival probabilities of the walkers. In addition to broken links and random measurements, traps corresponding to defects and impurities thus should be considered as a serious and additional source of decoherence in practical implementations of quantum random walks.

\section{ACKNOWLEDGMENTS}

M.G. acknowledges the travel support by DPT. Ö.E.M. gratefully acknowledges the hospitality and the support by the Dokuz Eylül University.
[1] S. Chandrasekhar, Rev. Mod. Phys. 15, 1 (1943).

[2] M. N. Barber and B. W. Ninham, Random and Restricted Walks: Theory and Applications (Gordon and Breach, New York, 1970).

[3] R. Motwani and P. Raghavan, Randomized Algorithms (Cambridge University Press, Cambridge, U.K., 1995).

[4] M. Jerrum and A. Sinclair, in Approximation Algorithm for NP-Hard Problems, edited by D. S. Hochbaum (PWS Publishing, Boston, 1996), Chap. 12, pp. 482-520.

[5] M. A. Nielsen and I. L. Chuang, Quantum Computation and Quantum Information (Cambridge University Press, Cambridge, England, 2000).

[6] D. Deutsch and R. Jozsa, Proc. R. Soc. London, Ser. A 439, 553 (1992).

[7] P. W. Shor, in Proceedings of the 35th Annual Symposium on the Foundations of Computer Science, edited by S. Goldwasser (IEEE Computer Society Press, Los Alamitos, CA, 1994), pp. 124-134.

[8] L. K. Grover, Phys. Rev. Lett. 79, 325 (1997).

[9] J. Kempe, Contemp. Phys. 44, 307 (2003).

[10] S. E. Venegas-Andraca, Quantum Walks for Computer Scientists (Morgan and Claypool Publishers, San Rafael, CA, 2008).

[11] N. Konno, in Quantum Potential Theory, Lecture Notes in Mathematics Vol. 1954, edited by U. Franz and M. Schürmann (Springer-Verlag, Heidelberg, 2008), pp. 309-452.

[12] A. M. Childs, R. Cleve, E. Deotto, E. Farhi, S. Gutmann, and D. A. Spielman, Proceedings of the 35th ACM Symposium on
Theory of Computing (ACM Press, New York, 2003), p. 59.

[13] N. Shenvi, J. Kempe, and K. Birgitta Whaley, Phys. Rev. A 67, 052307 (2003).

[14] A. M. Childs and J. Goldstone, Phys. Rev. A 70, 022314 (2004).

[15] Andris Ambainis, Julia Kempe, and Alexander Rivosh, Proceedings of the 16th ACM-SIAM SODA (Society for Industrial and Applied Mathematics, Philadelphia, PA, 2005), p. 1099.

[16] B. C. Travaglione and G. J. Milburn, Phys. Rev. A 65, 032310 (2002).

[17] W. Dur, R. Raussendorf, V. M. Kendon, and H. J. Briegel, Phys. Rev. A 66, 052319 (2002).

[18] K. Eckert, J. Mompart, G. Birkl, and M. Lewenstein, Phys. Rev. A 72, 012327 (2005).

[19] B. C. Sanders, S. D. Bartlett, B. Tregenna, and P. L. Knight, Phys. Rev. A 67, 042305 (2003).

[20] P. L. Knight, E. Roldán, and J. E. Sipe, Opt. Commun. 227, 147 (2003).

[21] B. Do, M. L. Stohler, S. Balasubramanian, D. S. Elliott, C. Eash, E. Fischbach, M. A. Fischbach, A. Mills, and B. Zwickl, J. Opt. Soc. Am. B 22, 499 (2005).

[22] P. K. Pathak and G. S. Agarwal, Phys. Rev. A 75, 032351 (2007).

[23] J. Du, H. Li, X. Xu, M. Shi, J. Wu, X. Zhou, and R. Han, Phys. Rev. A 67, 042316 (2003).

[24] C. A. Ryan, M. Laforest, J. C. Boileau, and R. Laflamme, Phys. Rev. A 72, 062317 (2005). 
[25] V. Kendon and B. Tregenna, Phys. Rev. A 67, 042315 (2003).

[26] V. Kendon, Math. Struct. Comp. Sci. 17, 1169 (2007).

[27] A. Romanelli, R. Siri, G. Abal, A. Auyuanet, and R. Donangelo, Physica A 347, 137 (2005).

[28] C. M. Chandrashekar, R. Srikanth, and Subhashish Banerjee, Phys. Rev. A 76, 022316 (2007).

[29] S. Havlin, G. H. Weiss, J. E. Kiefer, and M. Dishon, J. Phys. A 17, L347 (1984).

[30] S. Havlin, M. Dishon, J. E. Kiefer, and G. H. Weiss, Phys. Rev. Lett. 53, 407 (1984).

[31] P. Grassberger and I. Procaccia, J. Chem. Phys. 77, 6281 (1982).

[32] R. F. Kayser and J. B. Hubbard, Phys. Rev. Lett. 51, 79 (1983).

[33] H. E. Stanley, K. Kang, S. Redner, and R. L. Blumberg, Phys. Rev. Lett. 51, 1223 (1983).
[34] S. Redner and K. Kang, Phys. Rev. Lett. 51, 1729 (1983).

[35] M. A. Prasad and M. Nagarajan, J. Phys. A 32, 7665 (1999).

[36] K. Watabe, N. Kobayashi, M. Katori, and N. Konno, Phys. Rev. A 77, 062331 (2008).

[37] N. Inui, Y. Konishi, and N. Konno, Phys. Rev. A 69, 052323 (2004).

[38] T. D. Mackay, S. D. Bartlett, L. T. Stephenson, and B. C. Sanders, J. Phys. A 35, 2745 (2002).

[39] A. C. Oliveira, R. Portugal, and R. Donangelo, Phys. Rev. A 74, 012312 (2006).

[40] B. Tregenna, W. Flanagan, R. Maile, and V. Kendon, New J. Phys. 5, 83 (2003).

[41] R. Kohlrausch, Pogg. Ann. Phys. Chem. 91, 179 (1854).

[42] G. Williams and D. Watts, Trans. Faraday Soc. 66, 80 (1970).

[43] J. C. Phillips, Rep. Prog. Phys. 59, 1133 (1996). 